\title{
Immunosensor for okadaic acid using quartz crystal microbalance
}

\author{
Alice X J Tang ${ }^{\mathrm{a}, \mathrm{b}, *}$, Miloslav Pravda ${ }^{\mathrm{a}}$, George G Guilbault ${ }^{\mathrm{a}}$, Sergey Piletsky ${ }^{\mathrm{b}}$ \\ and Anthony P F Turner ${ }^{\mathrm{b}}$ \\ ${ }^{a}$ Chemistry Department, National University of Ireland, Cork; \\ ${ }^{b}$ Cranfield University at Silsoe, Bedfordshire MK45 4DT, UK
}

\begin{abstract}
An immunosensor for the determination of okadaic acid (OA) using a quartz crystal microbalance (QCM) was developed and optimised in standard solutions. Several coupling techniques, protein A, protein $\mathrm{G}$ and polyethylenimine (PEI) with glutaraldehyde (GA) crosslinking, were investigated for the determination of okadaic acid and a very good result was obtained with PEI coupling. With the PEI coupling method, the optimisation of incubation time for the activation of PEI on the crystal surface using GA, the effect of the dilution factor of OA-BSA conjugate and the amount of antibody on crystal frequency were studied. Different molar ratios $(4: 1,14: 1,30: 1)$ of OA to bovine serum albumin (BSA) for the conjugation were examined and the results using ELISA and a QCM showed that a ratio of 14:1 was slightly better than the other two. The strong attachment of the cross-linked complex to the gold surface resulted in an excellent storage lifetime of 38 days. However, the detection limit $(1.9 \mu \mathrm{g} / \mathrm{ml})$ and the sensitivity of the sensor were not satisfactory. Significant improvement of the performance of the device was obtained by incorporating an antibody-BSA hydrogel. Initial results showed that the minimum amount of analyte detectable and the sensitivity of the device were improved by 524 and 80 fold, respectively.
\end{abstract}

Keywords: quartz crystal microbalance, immunosensor, okadaic acid, anti-okadaic acid monoclonal antibody and antibody-BSA hydrogel.

* To whom correspondence should be addressed. 


\section{Introduction}

The quartz crystal microbalance is a label free technique, which minimises interference with the interaction being studied and also eliminates expensive and time-consuming purification procedures in many situations. A piezoelectric device is portable, simple and cost effective, and is suitable for real-time monitoring of biospecific interactions such as antigen-antibody, receptorligand, enzymes-substrate inetractions etc. with high sensitivity and specificity. The principles underlying measurement using a piezoelectric sensor and the general experimental apparatus were described by Luong and Guilbault [1]. The applications of quartz crystal microbalance immunosensors and future trends were presented in a review [2]. The first immunosensor based on QCM for detecting anti-BSA antibody by coating antigen was developed by Shons in 1972 [3]. Following this development, piezoelectric immunosensors have been widely used in liquid-phase analysis and have been adopted in the fields of clinical analysis, environmental monitoring [4], food analysis and veterinary diagnosis [5].

In general, protein immobilization on biosensor surfaces can be performed by covalent binding or adsorption or avidin-biotin binding on the crystal surface. For small ligands and proteins, covalent binding provides the highest irreversible surface loading and is therefore the best method to immobilize molecules on the crystal surface [6]. Interest in the properties and applications of covalent immobilisation based on self-assembled monolayers (SAMs) has grown enormously because they provide numerous advantages for sensor performance [7, 8]. Different types of covalent binding of materials on the surface of piezoelectric crystals were discussed in previous publications [9-11]. Adsorption procedures are easier and quicker than the other immobilization techniques, but they do not yield stable affinity systems for biosensor design. Weak bonds created by non-covalent attachment usually caurse severe leakage of the biomolecule off the surface and degradation of performance with use. Some of these methods can be problematic due to random orientation and detachment of the immobilized proteins [12]. The best immobilization technique depends on the individual system and it should provide good sensor performance in terms of sensitivity, selectivity, reproducibility and regeneration ability.

The objective of the present study was to develop a piezoelectric immunosensor for the determination of the algal toxin, okadaic acid (Figure 1). A competitive format (Figure 2) 
incorporating an immobilisation method using polyethylenimine with glutaraldehyde cross-linking was adopted. The crystal surface was modified with polymer polyethylenimine and the free amino groups obtained were activated by glutaraldehyde. OA-BSA conjugate was then bound to the activated derivatives to form a cross-linked complex, which strongly attached to the gold surface of the crystal, resulting in good long-term storage properties. However, the detection limit and the sensitivity of the sensor were not satisfactory. For this reason, an antibody-BSA hydrogel was adopted to improve the performance of the piezoelectric immunosensor. Stimuli-sensitive hydrogels have attracted considerable attention as intelligent materials, since they can abruptly change their volume in response to environmental change, such as solvent composition, $\mathrm{pH}$, temperature, concentration of guest, light and electric field [13-17]. Such gel swelling effects have been observed with both antibody and antigen systems [18]. This important phenomenon has not been previously applied to the detection of okadaic acid, a key algal toxin in the environment. Our studies commenced with an initial evaluation of these principles applied to the detection of OA. The work focused on the preparation of bovine serum albumin (BSA)-antibody hydrogel with glutaraledehyde cross-linking and the effect of concentration of okadaic acid on the volume of the gel.

\section{Experimental}

\subsection{Apparatus}

The liquid-phase piezoelectric detector and piezoelectric crystals (AT-cut, quartz plate diameter $14 \mathrm{~mm}$, gold electrode diameter $8 \mathrm{~mm}, 10 \mathrm{MHz}$ resonant frequency) used for the first phase of the work were purchased from Universal Sensors, INC (New Orleans, USA). For the work on the antibody-BSA gel, crystals with a $\mathrm{Cr} / \mathrm{Au}$ surface and a diameter of $6 \mathrm{~mm}$ were purchased from Technobiochip (Marciana Marina, Italy). ELISA was performed in a 96-well microtiter plate (Nalge Nunc International, Nunc-Immuno ${ }^{\mathrm{TM}}$ Plate, MaxiSorp ${ }^{\mathrm{TM}}$ Surface, made in Denmark). BioTek Instruments supplied the reader (model EL 311SX). A Slider-A-Lyzer cassette, 3,500 MWCO (from Pierce) was used for the purification of OA-BSA conjugate and the purified conjugate was analysed for protein content using an uv/vis spectrophotometer (Hewlett-Packard). 


\subsection{Chemicals}

Anti-okadaic acid monoclonal antibody was purchased from Iatron laboratories, INC (Tokyo, Japan). Okadaic acid (Prorocentrum concavum) was purchased from Calbiochem (Nottingham, UK), p-nitrophenyl phosphate (p-NPP), bovine serum albumin (BSA), 1-ethyl-3-(3dimethylaminopropyl) carbodiimide (EDC), N-hydroxy-succinimide (NHS), anti-mouse IgGalkaline phosphatase conjugate, dimethyl sulfoxide (DMSO), polyethylenimine (PEI), glutaraldehyde (GA) and other chemicals were obtained from Sigma Chemicals LTD (Dublin, Ireland).

\subsection{Conjugation of okadaic acid to bovine serum albumin}

Okadaic acid $(100 \mu \mathrm{g})$ was dissolved in $80 \mu \mathrm{l}$ of DMSO and added to the mixture of $10 \mu \mathrm{l}$ of EDC $(20 \mathrm{mg} / \mathrm{ml})$ and $10 \mu \mathrm{l}$ of NHS $(10 \mathrm{mg} / \mathrm{ml})$. The mixture was allowed to react for two hours by shaking gently at room temperature. A molar ratio of 14:1 was used in the conjugation of OA to BSA. BSA $(592 \mu \mathrm{g})$ was dissolved in $350 \mu \mathrm{l}$ of $0.1 \mathrm{M}$ phosphate buffer, $\mathrm{pH} 7.4$, containing $0.02 \%$ $\mathrm{N}_{\mathrm{a}} \mathrm{N}_{3}$ and was added drop-wise to activated-OA. The reaction was allowed to proceed for four hours by shaking gently on ice. After purification by dialysis with four changes of buffer overnight, the total volume of the conjugate was brought up to $1 \mathrm{ml}$ and the concentration of protein in the conjugate was determined at $280 \mathrm{~nm}$ using an uv/vis spectrophotometer. The molar absorptivity of BSA was estimated by measuring the absorbance of $1 \mathrm{mg} / \mathrm{ml} \mathrm{BSA}$ solution and was used to calculate the concentration of protein in OA-BSA.

\subsection{ELISA for the assessment of OA-BSA conjugate}

The immobilisation of OA-BSA conjugate onto a microtiter plate was achieved by adding $50 \mu 1$ of conjugation solution to each well and incubating, followed by blocking using $100 \mu \mathrm{l}$ of $1 \%$ BSA in $50 \mathrm{mM}$ Tris buffer, $\mathrm{pH}$ 7.4. The antibody binding was then carried out by adding $50 \mu \mathrm{l}$ of $30 \mu \mathrm{g}$ antibody solution and incubating, followed by the addition of $50 \mu \mathrm{l}$ of anti-mouse IgG- 
alkaline phosphatase (10,000-fold dilution) to each well. Colour development was achieved by the addition of $100 \mu \mathrm{l}$ of $1 \mathrm{mg} / \mathrm{ml}$-NPP in $0.1 \mathrm{M}$ diethanolamine, $\mathrm{pH} 9.5$ containing $1 \mathrm{mM} \mathrm{MgCl} 2$ and $50 \mathrm{mM} \mathrm{KCl}$ and incubating. The yellow product was monitored at $405 \mathrm{~nm}$. All incubations proceeded at $37{ }^{\circ} \mathrm{C}$ for one hour. Between incubations, the ELISA plate was washed three times with Tris buffer.

\subsection{Preparation of a piezoelectric sensor using polyethylenimine with cross-linking}

A crystal surface was cleaned as previously described in a reference [19]. The crystal was placed into a solution of $1.2 \mathrm{M} \mathrm{NaOH}$ for five minutes and then into $1.2 \mathrm{M} \mathrm{HCl}$ for five minutes, followed by the addition of $20 \mu \mathrm{l}$ of concentrated $\mathrm{HCl}$ for $1 \mathrm{~min}$. The crystal was then washed twice with ethanol and finally washed thoroughly with distilled water followed by drying at $37^{\circ} \mathrm{C}$.

PEI (10 $\mu \mathrm{l}$ of $2 \%$ in methanol) was added onto the crystal surface in a flow cell and after airdrying for $30 \mathrm{~min}$ the PEI-coated crystal was washed four times using methanol. The free amino group obtained on the crystal surface was activated using $40 \mu \mathrm{l}$ of $2.5 \%$ glutaraldehyde in distilled water and incubation proceeded for $45 \mathrm{~min}$. After washing thoroughly using distilled water, $15 \mu \mathrm{l}$ of a 1:2 dilution of stock OA-BSA conjugate plus $5 \mu \mathrm{l}$ of buffer was applied onto the treated crystal surface and allowed to react for $60 \mathrm{~min}$. After extensive washing, the crystal surface with cross-linked complex was blocked using $50 \mu \mathrm{l}$ of $0.1 \mathrm{M}$ glycine in $0.05 \mathrm{M}$ phosphate buffer, $\mathrm{pH} 7.4$ for 60 minutes. After washing and air-drying the device was stored dry in a fridge prior to use. All incubations proceeded at room temperature. To be able to cover the whole crystal surface a volume of $20 \mu \mathrm{l}$ solution was applied onto a crystal surface. The crystal frequency was monitored before (F1) and after (F2) material binding (non-real-time measurement), and the frequency change $(\Delta \mathrm{F})$ resulting from material binding was calculated using $\Delta \mathrm{F}=\mathrm{F}_{2}-\mathrm{F}_{1}$.

For the calibration for okadaic acid, a mixture of free OA $(5 \mu \mathrm{l})$ and $3.75 \mu \mathrm{g}$ of $\alpha$-OA $(15 \mu \mathrm{l}$, $0.25 \mu \mathrm{g} / \mu \mathrm{l})$ was incubated for $20 \mathrm{~min}$ before adding to a sensor surface. After the incubation of the mixture onto the sensor surface for $60 \mathrm{~min}$ the sensor surface was washed twice using $200 \mu \mathrm{l}$ of distilled water and its frequency was monitored.

\subsection{Preparation of an antibody-BSA hydrogel on a crystal surface}


A crystal was placed into a solution of $\mathrm{H}_{2} \mathrm{O}_{2} / \mathrm{H}_{2} \mathrm{SO}_{4}(1: 3, \mathrm{~V} / \mathrm{V})$ for 10 seconds and washed thoroughly using distilled water. After drying in an oven it was stored in a desiccator for up to one week. The procedure for the preparation of the membrane was similar to the method previously described by Wan [20]. Antibody solution $(200 \mu \mathrm{l}, 5 \mathrm{mg} / \mathrm{ml})$ was freeze dried and was then dissolved in $20 \mu \mathrm{l}$ of $5 \%$ BSA solution in $0.05 \mathrm{M}$ phosphate buffer, $\mathrm{pH} 7$, containing $5 \%$ glycerol. An aliquot of the mixture $(3 \mu \mathrm{l})$ was applied onto a clean crystal surface and the crystal was placed in a saturated glutaraldehyde vapour to form a solid membrane by cross-linking. In order to protect the electrical connections of the crystal they were covered with adhesive tape during the exposure to glutaraldehyde. The sensor was allowed to form a solid membrane on its surface while stored in a refrigerator $\left(4{ }^{\circ} \mathrm{C}\right)$ overnight. The glycerol served as a plasticizer to inhibit the formation of cracks in the membrane and this resulted in a homogenous membrane and better adhesion to the gold surface. The sensors were stored dry in a fridge prior to use.

\subsection{Measurement system for crystal with an antibody-BSA gel}

Measurements were carried out with a single-channel flow injection system assembled with Teflon tubing and a peristaltic pump (Gilson Minipuls 3, Paris, France) with a flow rate of 20 $\mu 1 /$ min. The coated crystal was mounted in a flow cell which was connected to a Libra $\alpha$ Nanobalance Measuring System (Technobiochip, Marciana, Italy) to record the frequency. The Libra $\alpha$ Nanobalance was interfaced to a PC for data recording and processing. The system was driven by LIBRA 3.1 software running under MS-Windows ${ }^{\mathrm{TM}}$. Before making an injection the sensor was stabilized by running $0.05 \mathrm{M}$ phosphate buffer, $\mathrm{pH}$ 7.0, for about 6 hours. Sample solution $(500 \mu \mathrm{l})$ containing okadaic acid in the same buffer, was introduced into the buffer stream via a valve loop injector. The oscillation frequency of crystal was recorded with time (real-time measurement) and the frequency change $(\Delta \mathrm{F})$ resulting from the reaction of the antibody with the antigen was calculated from the difference in frequency before $\left(\mathrm{F}_{1}\right)$ and after $\left(\mathrm{F}_{2}\right)$ the binding event, $\Delta \mathrm{F}=\mathrm{F}_{2}-\mathrm{F}_{1}$.

\section{Results and discussion}




\subsection{Investigation of a coupling method for detecting okadaic acid}

Four crystals were taken as supplied, rinsed with distilled water and then dried in air for 35 minutes. Considerable decreases in oscillation frequency of the crystals were observed due to water variable retention. To eliminate this effect on a crystal frequency and avoid air-drying step $100 \mu \mathrm{l}$ of distilled water was added onto each crystal surface prior to frequency measurement before and after biomolecule binding.

The determination of okadaic acid using a piezoelectric immunosensor with different coupling methods was investigated. The techniques examined were protein $A$, protein $G$ and polyethylenimine with glutaraldehyde cross-linking. With protein A coupling, buffer $\mathrm{pH}$ values of 5.5 and 7.4 for protein A binding and $\mathrm{pH}$ values of 5.5, 7.4 and 9.6 for antibody binding were tested and the results showed that the best immobilisation occurred at $\mathrm{pH}$ of 5.5 and 7.4 for the protein A binding and for the antibody binding, respectively. The frequency change resulting from anti-OA antibody $(1 \mathrm{mg} / \mathrm{ml})$ binding using protein $\mathrm{A}$ and protein $\mathrm{G}$ were similar. However, the signal obtained in the determination of okadaic acid was very small. A large frequency change for OA-BSA binding to a gold surface was obtained using polyethylenimine with glutaraldehyde cross-linking, and therefore this method was applied to our further studies.

\subsection{Characterisation}

In order to provide an immunosensor with a good performance the molar ratios of OA:BSA for the conjugatation, the incubation time for the activation of PEI on a crystal surface using glutaraldehyde, the influence of the dilution factor of the conjugate and the amount of antibody on a crystal frequency were investigated.

Different molar ratios (4:1, 14:1 and 30:1) of OA:BSA were studied for the conjugation of OA to BSA. The results with ELISA showed that the absorbance for the conjugate (14:1) was 1.5 times higher than the others. The two conjugates (14:1 and 30:1) were also used with piezoelectric crystals and the frequency changes for both conjugates were almost same. The molar ratio of 14:1 in the conjugation of OA to BSA was adopted in further work. 
Optimisation of the incubation time for the activation of PEI on a crystal surface using glutaraldehyde was performed. Figure 3 shows that the frequency change of a crystal increased with the incubation time of glutaraldehyde on PEI-coated crystals and reached a plateau at incubation time of $45 \mathrm{~min}$.

The effect of the dilution factor of OA-BSA conjugate on crystal frequency was investigated. The dilution factors $(1: 8,1: 4$ and 1:2) of OA-BSA conjugate were examined and the results obtained were shown in Figure 4. The dilution factor of 1:2 of conjugate produced an adequate signal and it was adopted for further work.

As expected, the frequency change of sensors increased with increasing amount of antibody added onto their surfaces. As shown in Figure 5,20 $\mu \mathrm{g}$ of antibody $(20 \mu \mathrm{l}, 1.0 \mu \mathrm{g} / \mu \mathrm{l}$ of antibody) produced a satisfactory response with a frequency change of $440 \mathrm{~Hz}$ being recorded. In order to minimise the amount of antibody needed to produce a significant signal, improvement of the quality of the OA-BSA conjugate was studied. The results obtained showed that significantly less antibody was required to generate a response; $3.75 \mu \mathrm{g}$ of antibody resulted in a frequency change of approximately $500 \mathrm{~Hz}$ when using the new OA-BSA conjugate, this was because a higher density of conjugate (more okadaic acid bound to the BSA) was obtained by using a more concentrated BSA solution for the conjugation.

\subsection{Storage lifetime of a piezoelectric immunosensor}

Clean crystals were treated with polyethylenimine, glutaraldehyde and OA-BSA conjugate and were then blocked using glycine as described in the experimental section. After air-drying the sensors were stored dry in a refrigerator $\left(4^{\circ} \mathrm{C}\right)$. The frequencies of three treated crystals were measured regularly over a period of one week and no changes in frequency were observed (Figure 5). The immobilisation of antibody $(15 \mu \mathrm{l}, 0.5 \mu \mathrm{g} / \mu \mathrm{l})$ onto the sensor's surface with time was further investigated at 1, 19 and 39-days after storage. The results obtained showed that after 38 days the immobilization of antibody to the sensor's surface produced the same frequency change as that obtained with the freshly treated sensors. This good long-term storage stability allowed us to prepare many sensors in a single batch thus aiding consistency between experiments and saving materials and time. 


\subsection{Calibration for okadaic acid using polyethylenimine with cross-linking}

Since okadaic acid dissolves in organic solvents such as ethanol and a quartz crystal senses the density change on its surface, the effect of ethanol on a crystal surface was studied with both realtime (crystal frequency was measured versus reaction time) and non-real-time measurement as mentioned in the experimental section. The results obtained showed that the crystal frequency decreased on the addition of ethanol to distilled water on sensors surface with real-time measurement, but a change in frequency was not observed in the case of the non-real-time measurement. Therefore the non-real-time measurement mode was applied to produce the calibartion curve for okadaic acid to avoid the effect of ethanol on the oscillation frequency of the crystal.

Different incubation times ( 0 and $20 \mathrm{~min})$ of the mixture of antibody and free okadaic acid were tested prior to application to the sensor. Following incubation of the mixture for zero minutes, about $63 \%$ of antibody was bound to OA-BSA conjugate immobilized on the crystal surface after one hour reaction time between the mixture and conjugate. This resulted in less competition between free $\mathrm{OA}$ and $\mathrm{OA}$ in conjuate on the crystal surface. In contrast, at the mixture incubation time of 20 min most of antibody reacted with the free okadaic acid and much less antibody was bound to OA-BSA conjugate on the sensor surface, resulting in a higher sensitivity to okadaic acid.

The calibration curve for okadaic acid was prepared in the concentration range from 0 to 25 $\mu \mathrm{g} / \mathrm{ml}$ of okadaic acid and the linear portion is shown in Figure $\underline{6}$ (two crystals were used for each point on the curve), and it was linear up to $16 \mu \mathrm{g} / \mathrm{ml}$ with a detection limit of $1.9 \mu \mathrm{g} / \mathrm{ml}$. An immunosensor for okadaic acid based on the technique of screen-printing has been previously reported by Tang [21] and the detection limit and the sensitivity were orders of magnitude better than piezoelectric immunosensor. To produce a satisfactory device for okadaic acid an attempt was made to improve the performance of a piezoelectric immunosensor for okadaic acid by using an antibody-BSA gel.

\subsection{Improvement in sensitivity of piezoelectric sensor using an antibody-BSA hydrogel}


Three batches of crystals with antibody-BSA gels were made and it was observed that antibodyBSA membranes for the first batch detached partially from crystal surfaces after measurements. This can be explained by the fact that the antibody and BSA were not completely cross-linked when the sensors were placed in unsaturated glutaraldehyde vapour. Subsequent results showed that a saturated glutaraldehyde vapour resulted in good adhesion of the membrane to the gold surfaces of the crystals.

Five crystals were prepared with anti-okadaic acid antibody and bovine serum albumin gel according to the immobilization procedures described above. The sensors were evaluated in a flow injection system and the decreases in frequency were observed when okadaic acid solution was introduced in the buffer flow. Each sensor could be used for a couple of measurements. A typical set of measurements for one crystal with three different concentrations of analyte $(\underline{10,25}$ and 50 $\mathrm{ng} / \mathrm{ml}$ ) showed that the frequency change of the sensor increased with the concentration of okadaic acid and reached a plateau at $50 \mathrm{ng} / \mathrm{ml}$ of okadaic acid. By comparison, the lowest measurable concentration of okadaic acid and the sensitivity of the sensor were improved by 524 and 80 fold, respectively. A crystal with BSA gel only was studied as a control in the determination of okadaic acid. Decrease in frequency of the crystal was initially observed, but the oscillation frequency returned to the baseline after continuously introducing the buffer flow. This phenomenon in the control crystal can be explained by the fact that the oscillation frequency of crystal changed in response to the different composition of the sample solution in the buffer flow.

Since a quartz crystal microbalance conventionally senses the mass change on its surface and okadaic acid is a small molecule, it is unlikely that direct binding of okadaic acid to immobilised antibody would result in a good detection limit and high sensitivity. We therefore conclude that the oscillation frequency of crystals with hydrogel decreased due to a volume change of the gel resulting from the binding of okadaic acid to antibody recognition sites.

\section{Conclusions}

The quartz crystal microbalance using polyethylenimine coupling with glutaraldehyde crosslinking in conjunction with the competitive format was able to quantify okadaic acid in standard 
solution. The polyethylenimine immobilisation with glutaraldehyde cross-linking was a successful coupling method that produced a piezoelectric immunosensor with a good long-term storage lifetime of 38 days. However, the sensitivity of the device for okadaic acid was not satisfactory.

The antigen-sensitive hydrogel proved to be a useful approach for the development of a highly sensitive quartz crystal microbalance for okadaic acid. Initial results were very promising, demonstrating orders of magnitude improvement in both the minimum amount of analyte detectable and the sensitivity of the device. These improvements could pave the way for a practical piezoelectric immunosensor for environmental monitoring. Naturally, further optimisation is required to improve performance and to demonstrate the practical usefulness of this new approach for okadaic acid in mussels.

\section{Acknowledgements}

The authors would like to express their gratitude for the financial support from EU (QLKI-CT1999-51197) and the EU Thematic Network SENSPOL (EVKI-CT-1999-20001).

\section{References}

[1] J. Luong and G.G. Guilbault, In: Biosensor Principles and Applications, edited by L.. J.

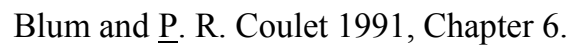

[2] C.K. O’Sullivan and G.G. Guilbault, Biosens. Bioelectron., 14 (1999) 663.

[3] A. Shons, F. Dorman and J. Najarian, J. Biomed. Mater. Res., 6 (1972) 565.

[4] K. Yokoyama, K. Ikebukuro, E. Tamiya, I. Karube, N. Ichiki and Y. Arikawa, Anal. Chim. Acta, 304 (1995) 139.

[5] X.D. Su, S.F.Y. Li, W. Liu and J. Kwang, Anal., 125 (2000) 725.

[6] R. Ulbrich, R. Golbik and A. Achellenberger, Biotechnol. Bioeng., 37 (1991) 280.

[7] A. Ulman, An Introduction to Ultrathin Organic Film, Academic Press, San Diego, CA, Part III, and reference cited therein, 1991.

[8] J. Rickert, A. Brecht and W. Gopel, Biosens. Bioelectron., 12 (1997) 567.

[9] S. Storri, T. Santoni, M. Minunni and M. Mascini, Biosens. Bioelectron., 13 (1998) 347. 
[10] G.B. Sigal, C. Bamdad, A. Barberis, J. Strominger and G.M. Whitesides, Anal. Chem., 68 (1996) 490.

[11] C. Steegborn and P. Skládal, Biosens. Bioelectron., 12 (1997) 19.

[12] S. Kurosawa, E. Tawara, N. Kamo, F. Ohta and T. Hosokawa, Chem. Pharm. Bull (Jpn), 38 (1990) 1117.

[13] T. Tanaka, Phys. Rev. Lett., 40 (1978) 820.

[14] T. Tanaka, D. Fillmore, S.T. Sun, I. Nishio, G. Swislow and A. Shah, Phys. Rev. Lett., 45 (1980) 1636.

[15] T. Takana, I. Nishio, S.T. Sun and S. Ueno-Nishio, Sci., 218 (1982) 467.

[16] M. Irie and D. Kungwatchakun, Macromol. Chem. Rapid. Commun., 5 (1984) 829.

[17] M. Watanabe, T. Akahoshi, Y. Tabata and D. Nakayama, J. Am. Chem. Soc., 120 (1998) 5577.

[18] T. Miyata, N. Asami and T. Uragami, Nature, 399 (1999) 766.

[19] K.A. Davis and T. Leary, Anal. Chem., 61 (1989) 1227.

[20] K. Wan, J.M. Chovelon, N. Jaffrezic-R. and A.P. Soldatkin, Sens. Actuat., 58 (1999) 399.

[21] A. Tang, M. Kreuzer, M. Lehane, M. Pravda and G.G. Guilbault, J. Environ. Anal. Chem., 2002 (in press). 


\section{Figure captions}

Fig. 1. Chemical structure of okadaic acid.

Fig. 2. Schematic diagram of competitive format.

Fig. 3. Optimisation of incubation time for the activation of PEI on crystal surface using glutaraldehyde. The OA-BSA was a two-fold dilution of stock conjugate. The relative frequency change $(\Delta \mathrm{F})$ is normalised; Relative $\Delta \mathrm{F}=($ actual $\Delta \mathrm{F} /$ maximum $\Delta \mathrm{F}) \times 100$.

Fig. 4. Frequency change of crystal with dilution factor of OA-BSA conjugate. Molar ratio of OA:BSA for the preparation of the conjugate was 14:1 and the concentration of protain in OABSA was $0.374 \mu \mathrm{g} / \mu \mathrm{l}$.

Fig. 5. Investigation of the frequency response of three sensors with cross-linked complex over a period of one-week's storage in a refrigerator $\left(4^{\circ} \mathrm{C}\right)$.

Fig. 6. Calibration curve for okadaic acid with immobilisation using polyethylenimine with glutaraldehyde cross-linking using competitive assay. $3.75 \mu \mathrm{g}(15 \mu \mathrm{l}, 0.25 \mu \mathrm{g} / \mu \mathrm{l})$ of antibody was applied. The concentration of protein in OA-BSA conjugate was $0.554 \mu \mathrm{g} / \mu \mathrm{l}$. 